\title{
Elimination of high-refined-sugar diet as treatment strategy for autistic features induced in a rodent model
}

\author{
Sooad Al-Daihan ${ }^{1}$, Ramesa S Bhat ${ }^{1 *}$, Abeer M Al Dbass ${ }^{1}$, Laila Al-Ayadhi ${ }^{3-5}$ and \\ Afaf El-Ansary ${ }^{2-4}$ \\ ${ }^{1}$ Biochemistry Department, Science College ${ }^{2}$ Central Laboratory. Female Center for Medical Studies and Scientific Section, \\ ${ }^{3}$ Autism Research and Treatment Center, ${ }^{4}$ Shaik Al-Amodi Autism Research Chair, ${ }^{5}$ Department of Physiology, Faculty of \\ Medicine, King Saud University, Riyadh, Saudi Arabia
}

*For correspondence: Email: rbhat@ksu.edu.sa; Tel: 009661-4682184

Sent for review: 7 January 2016

Revised accepted: 14 June 2017

\begin{abstract}
Purpose: To investigate the potency of ampicillin in altering gut flora in the presence of a high-sucrose diet in rat pups, and to determine its effect on selected neurotransmitters and a cytokine as markers of the persistent autistic features repeatedly induced in orally administered propionic acid rat pups..

Methods: Twenty-eight young male Wistar albino rats were divided into four equal groups. The first group served as a control. The second group received an oral neurotoxic dose of propionic acid (PPA, $250 \mathrm{mg} / \mathrm{kg}$ body weight/day) for 3 days. The third group was treated with ampicillin (50 mg/kg for 3 weeks) with a standard diet. The fourth group was given the same dose of ampicillin with a high-sucrose diet for 10 weeks.

Results: The results showed a significant $(p<0.001)$ decrease in the investigated neurotransmitters in PPA- and ampicillin-treated rat pups (norepinephrine by 32.49 and $14.58 \%$, dopamine by 31.45 and $20.22 \%$, serotonin by 35.99 and $29.09 \%)$, as well as a remarkable increase $(p<0.001)$ in the proinflammatory cytokine, IL-6 (30.07and $6.07 \%)$. The high-sucrose diet also significantly $(p<0.001)$ enhanced the neurotoxic effect of ampicillin.

Conclusion: The observed dietary modulation of the gut microbiota, coupled with the subsequent modulation of brain neurochemistry and inflammation, demonstrates the considerable potential of dietary intervention through the elimination of highly refined sugar as a treatment strategy to prevent and treat autism.
\end{abstract}

Keywords: Neurotoxicity, Ampicillin, Propionic acid, Neurotransmitters, Cytokines, High-sucrose diet

Tropical Journal of Pharmaceutical Research is indexed by Science Citation Index (SciSearch), Scopus, International Pharmaceutical Abstract, Chemical Abstracts, Embase, Index Copernicus, EBSCO, African Index Medicus, JournalSeek, Journal Citation Reports/Science Edition, Directory of Open Access Journals (DOAJ), African Journal Online, Bioline International, Open-J-Gate and Pharmacy Abstracts

\section{INTRODUCTION}

Antibiotics are prescribed frequently for infants and children in nearly all pediatric clinics [1].Antibiotic treatment over a long period results in a 10-fold increase of Clostridium spp (propionibacteria) in the gut. Overgrowth of this species has also been reported in autistic patients [2].Furthermore, the induction of persistent autistic features in rodent modelswas achieved by MacFabe et al [3] and El-Ansary et al [4] through propionic acid (PPA) exposure. A concomitant increase in the fecal concentrations of PPA in children with autism may indicate the role by which microbial metabolites alter brain function through the gut-brain axis. It is now acknowledged that the gut-brain axis and balanced gut microbiota are critically important during brain development and can play a major role in the pathogenesis of autism. In the gut, several members of the Bifidobacterium and Lactobacillus genera have been found to 
possess health-promoting properties and are affected by diet, whereas many Proteobacteria have the potential to become pathogenic when a suitable environment is provided. Due to immature renal function and blood brain barrier, ampicillin is neurotoxic in very low birth weight neonates, resulting in a remarkable increase of ampicillin in both serum and CSF [5]. Ampicillin is believed to exert an inhibitory effect on GABA transmission [6]. Imbalances in GABAergic/glutamatergic, serotonergic, and dopaminergic neurotransmission together with neuroinflammation were recently reported as the most important signals related to the clinical presentation and severity of autism [7].

This information motivated our interest to compare the gut-brain neurotoxic effect of ampicillin to that induced by orally administered PPA as a metabolic end-product of propionibacteria. The impact of a high-refinedsugar diet on the neurochemical effects induced by ampicillin was also assessed.

\section{EXPERIMENTAL}

\section{Animals}

The study included 28 male Wistar albino rats divided into four independent groups containing seven rats each. Group I served as the control and received only phosphate buffered saline; Group II was administered with PPA (250 mg/kg body weight/day) for three days [4]; Group III was treated with an oral dose of ampicillin $(50 \mathrm{mg} / \mathrm{kg}$ for three weeks) with a standard diet; and Group IV was given the same dose of ampicillin with a high-sucrose diet for 10 weeks. The study was approved by the Ethics Committee of King Saud University with approval no.KSU-IRB008E. International animal care guidelines were strictly followed [8].

\section{Diet}

The special diet characterized by high sugar was made by mixing sucrose with regular chow.

\section{Sample preparation}

Brain tissue was homogenized in 10 times w/v bidistilled water and was used to measure all parameters evaluated in the study.

\section{Assay for neurotransmitters (NA, DA and 5HT)}

High-performance liquid chromatography with electrochemical detection was used to measure dopamine, serotonin and norepinephrine [9].

\section{IL-6 assay}

IL-6 was assayed using a Quantikine ELISA kit ( $R$ \& D Systems, Minneapolis, MN, USA). A microplate was pre-coated with a monoclonal antibody specific for rat IL-6.

\section{Glutamine and glutamate assays}

Rat brain glutamine and glutamate were measured independently using Cusabio ELISA Kits.

\section{Microbiological examination}

Fecal samples were used to identify the gut bacteria by culturing the samples under aerobic and anaerobic conditions. All the species found were identified using Scepter micro dilution and standard bacteriological techniques.

\section{Statistical analysis}

SPSS (Chicago,IL, USA) was used to analyze the data by using ANOVA for one-way analysis of variance and Dunnett test for multiple comparisons. $\mathrm{P}$ values less than 0.05 were considered significant. Receiver operating characteristics curve (ROC) analysis was performed to test the predictive value of the investigated parameters and to measure the toxicity of PPA and ampicillin in intoxicated rats. An area under the curve (AUC) of 0.8-1 indicated an excellent predictive value for the biomarker. This was accompanied by satisfactory values for sensitivity and specificity. Pearson's correlation analysis was also performed using the same SPSS program. Multiple regression analysis was conducted using glutamate and IL-6 as two dependent variables against the rest of the variables, which were used as independent or predictor variables.

\section{RESULTS}

Ampicillin treatment for six weeks altered the gut flora, resulting in unusual growth of a few PPA producers (e.g., Klebsiella pneumonia and Proteus vulgaris), while some species, such as Enterobacter cloacae, vanished. Ampicillin also promoted the overgrowth of Candida albicans.

Table 1 and Figure 1 show the significant changes in the brain chemistry of rats treated with PPA and ampicillin, as well as changes in those fed a high sucrose diet for 10 weeks post antibiotic treatment. NA was significantly reduced in both PPA and ampicillin-treated groups by $32.49 \%$ and $14.58 \%$, respectively. However, the high-sucrose diet in ampicillin-treated rats was 
the most potent, as it induced a $43.11 \%$ decrease in NA in the brain. The three groups differed from the control $(p<0.001)$. However, PPA induced the most significant decrease in DA and $5 \mathrm{HT}$.

Regarding the excitotoxicity presented by glutamate, PPA was the most potent, as it induced a $56.3 \%$ increase, followed by the other two groups, which demonstrated a 23.55 and $17.75 \%$ increase, respectively. The glutamine level was equally affected in the different treatments, showing an approximately $35 \%$ increase at $p<0.013$. The glutamate/glutamine ratio showed a non-significant difference compared to the control (Figure 1 and Table 2).

IL-6 was remarkably elevated in all experimental groups compared to that of the control. PPA and a HSD induced similar increases in IL-6 (30\% and $27 \%$, respectively), which were noticeably higher than that in ampicillin-treated animals $(6.07 \%)$ (Table 1 and Figure 1).

Table 1: Biochemical parameters of PPA, ampicillin-treated and ampicillin/HSD-fed rats

\begin{tabular}{|c|c|c|c|c|c|}
\hline Parameter & Group & $\mathbf{N}$ & Mean \pm S.D. & $\begin{array}{l}\text { Percent } \\
\text { change }\end{array}$ & $P$-value \\
\hline \multirow{4}{*}{ NA } & Control & 7 & $6.92 \pm 0.78$ & 100.00 & \multirow{4}{*}{0.001} \\
\hline & PPA & 7 & $4.67 \pm 0.45$ & 67.51 & \\
\hline & Ampicillin & 7 & $5.91 \pm 0.28$ & 85.42 & \\
\hline & HSD & 7 & $3.93 \pm 0.45$ & 56.89 & \\
\hline \multirow{4}{*}{ DA } & Control & 7 & $25.95 \pm 1.68$ & 100.00 & \multirow{4}{*}{0.001} \\
\hline & PPA & 7 & $17.79 \pm 1.34$ & 68.55 & \\
\hline & Ampicillin & 7 & $20.70 \pm 3.37$ & 79.78 & \\
\hline & HSD & 7 & $21.46 \pm 0.78$ & 82.72 & \\
\hline \multirow{4}{*}{ IL-6 } & Control & 7 & $227.34 \pm 19.47$ & 100.00 & \multirow{4}{*}{0.001} \\
\hline & PPA & 7 & $295.71 \pm 23.92$ & 130.07 & \\
\hline & Ampicillin & 7 & $241.15 \pm 23.26$ & 106.07 & \\
\hline & HSD & 7 & $290.22 \pm 41.24$ & 127.66 & \\
\hline \multirow{4}{*}{$5-\mathrm{HT}$} & Control & 7 & $8.59 \pm 0.42$ & 100.00 & \multirow{4}{*}{0.001} \\
\hline & PPA & 7 & $5.50 \pm 0.66$ & 64.01 & \\
\hline & Ampicillin & 7 & $6.02 \pm 0.51$ & 70.10 & \\
\hline & HSD & 7 & $6.03 \pm 0.55$ & 70.25 & \\
\hline \multirow{4}{*}{ Glut } & Control & 7 & $237.99 \pm 18.93$ & 100.00 & \multirow{4}{*}{0.007} \\
\hline & PPA & 7 & $371.97 \pm 105.29$ & 156.30 & \\
\hline & Ampicillin & 7 & $294.03 \pm 57.76$ & 123.55 & \\
\hline & HSD & 7 & $280.22 \pm 47.12$ & 117.75 & \\
\hline \multirow{4}{*}{ Gln } & Control & 7 & $2169.73 \pm 223.94$ & 100.00 & \multirow{4}{*}{0.013} \\
\hline & PPA & 7 & $2930.67 \pm 664.56$ & 135.07 & \\
\hline & Ampicillin & 7 & $2921.69 \pm 520.35$ & 134.66 & \\
\hline & HSD & 7 & $2967.29 \pm 412.29$ & 136.76 & \\
\hline \multirow{4}{*}{ Glut/ Gln } & Control & 7 & $9.15 \pm 1.10$ & 100.00 & \multirow{4}{*}{0.183} \\
\hline & PPA & 7 & $8.36 \pm 2.80$ & 91.35 & \\
\hline & Ampicillin & 7 & $10.15 \pm 2.02$ & 110.89 & \\
\hline & HSD & 7 & $10.82 \pm 2.37$ & 118.24 & \\
\hline
\end{tabular}

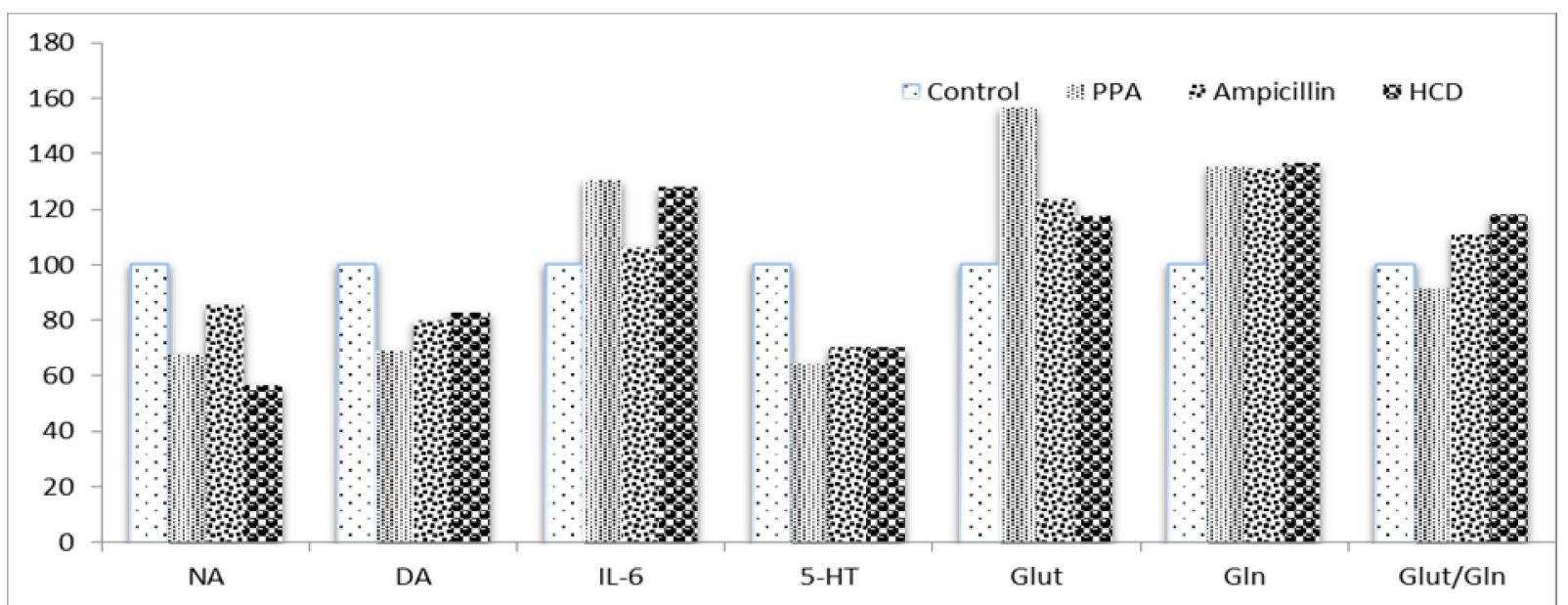

Figure 1: Percent change in all measured parameters 
Table 2: Pearson's correlation between the measured parameters

\begin{tabular}{lcc}
\hline Parameter & $\begin{array}{c}\text { R (Pearson's } \\
\text { correlation) }\end{array}$ & P value \\
\hline NA DA & 0.496 & 0.007 \\
NA IL-6 & -0.645 & 0.001 \\
NA 5-HT & 0.678 & 0.001 \\
NA Gln & -0.556 & 0.002 \\
DA IL-6 & -0.563 & 0.002 \\
DA 5-HT & 0.756 & 0.001 \\
DA Glut & -0.508 & 0.006 \\
DA Gln & -0.509 & 0.006 \\
IL-6 5-HT & -0.610 & 0.001 \\
IL-6 Gln & 0.563 & 0.002 \\
IL-6 Glut/Gln & 0.374 & 0.050 \\
5-HT Glut & -0.466 & 0.012 \\
5-HT Gln & -0.639 & 0.001 \\
Glut Glut/Gln & -0.592 & 0.001 \\
Gln Glut/Gln & 0.545 & 0.003 \\
\hline
\end{tabular}

Table 2 presents the Pearson's correlations between the measured parameters. It is clear that the neuroinflammatory marker IL-6 was positively correlated with glutamine and glutamate/glutamine ratio and was negatively correlated with NA, DA and 5HT. In addition, NA, DA and $5 \mathrm{HT}$ were positively correlated with each other and negatively correlated with glutamate, glutamine and glutamate/glutamine ratio.

Table 3 and Figure 2 present the area under the curve (AUC), specificity and sensitivity for the measured neurotransmitters and for IL-6 in the studied groups.

Table 4 shows the results of multiple regression analysis using glutamate as a dependent variable. It can be easily confirmed that glutamate is related to glutamine and the glutamate/glutamine ratio, recording $R^{2}$ values of 0.896 .

\section{DISCUSSION}

As gastrointestinal conditions and eating problems are common in autism, diet might represent a link between environmental and neurobiological factors and thus may play a role in the pathways leading to the etiology of this disorder. The impact of PPA, ampicillin treatment and a high-sucrose diet on the alteration of brain chemistry will be discussed. The recorded overgrowth of Candida albicans in the gut can be easily related to the induced autistic features. Yeast feed on sugar and produce alcohol, a toxic chemical that can easily be transported in the blood to the frontal lobes of the brain, where it

Table 3: ROC analysis of the measured parameters demonstrating area under the curve (AUC), cutoff values, sensitivity and specificity in the three experimental groups.

\begin{tabular}{llcccc}
\hline Variable & Group & $\begin{array}{c}\text { Area under } \\
\text { the curve }\end{array}$ & $\begin{array}{c}\text { Cutoff } \\
\text { value }\end{array}$ & $\begin{array}{c}\text { Sensitivity } \\
(\%)\end{array}$ & $\begin{array}{c}\text { Specificity } \\
(\%)\end{array}$ \\
\hline \multirow{3}{*}{ NA } & PPA & 1.000 & 5.420 & 100.0 & 100.0 \\
& Ampicillin & 0.918 & 6.365 & 100.0 & 85.7 \\
DA & HCD & 1.000 & 5.220 & 100.0 & 100.0 \\
& PPA & 1.000 & 21.950 & 100.0 & 100.0 \\
IL-6 & Ampicillin & 0.918 & 23.675 & 85.7 & 100.0 \\
& HCD & 1.000 & 23.435 & 100.0 & 100.0 \\
& PPA & 1.000 & 258.510 & 100.0 & 100.0 \\
5-HT & Ampicillin & 0.673 & 252.070 & 42.9 & 100.0 \\
& HCD & 0.959 & 252.260 & 85.7 & 100.0 \\
Glut & PPA & 1.000 & 7.115 & 100.0 & 100.0 \\
& Ampicillin & 1.000 & 7.300 & 100.0 & 100.0 \\
\multirow{3}{*}{ Gln } & HCD & 1.000 & 7.380 & 100.0 & 100.0 \\
& PPA & 1.000 & 267.650 & 100.0 & 100.0 \\
& Ampicillin & 0.878 & 257.650 & 85.7 & 85.7 \\
Glut/Gln & HCD & 0.816 & 258.140 & 71.4 & 85.7 \\
& PPA & 0.918 & 2551.090 & 71.4 & 100.0 \\
& Ampicillin & 0.959 & 2517.300 & 85.7 & 100.0 \\
& HCD & 1.000 & 2514.155 & 100.0 & 100.0 \\
& PPA & 0.612 & 7.436 & 42.9 & 100.0 \\
& Ampicillin & 0.694 & 9.185 & 71.4 & 71.4 \\
& HCD & 0.755 & 9.385 & 85.7 & 71.4 \\
\hline
\end{tabular}



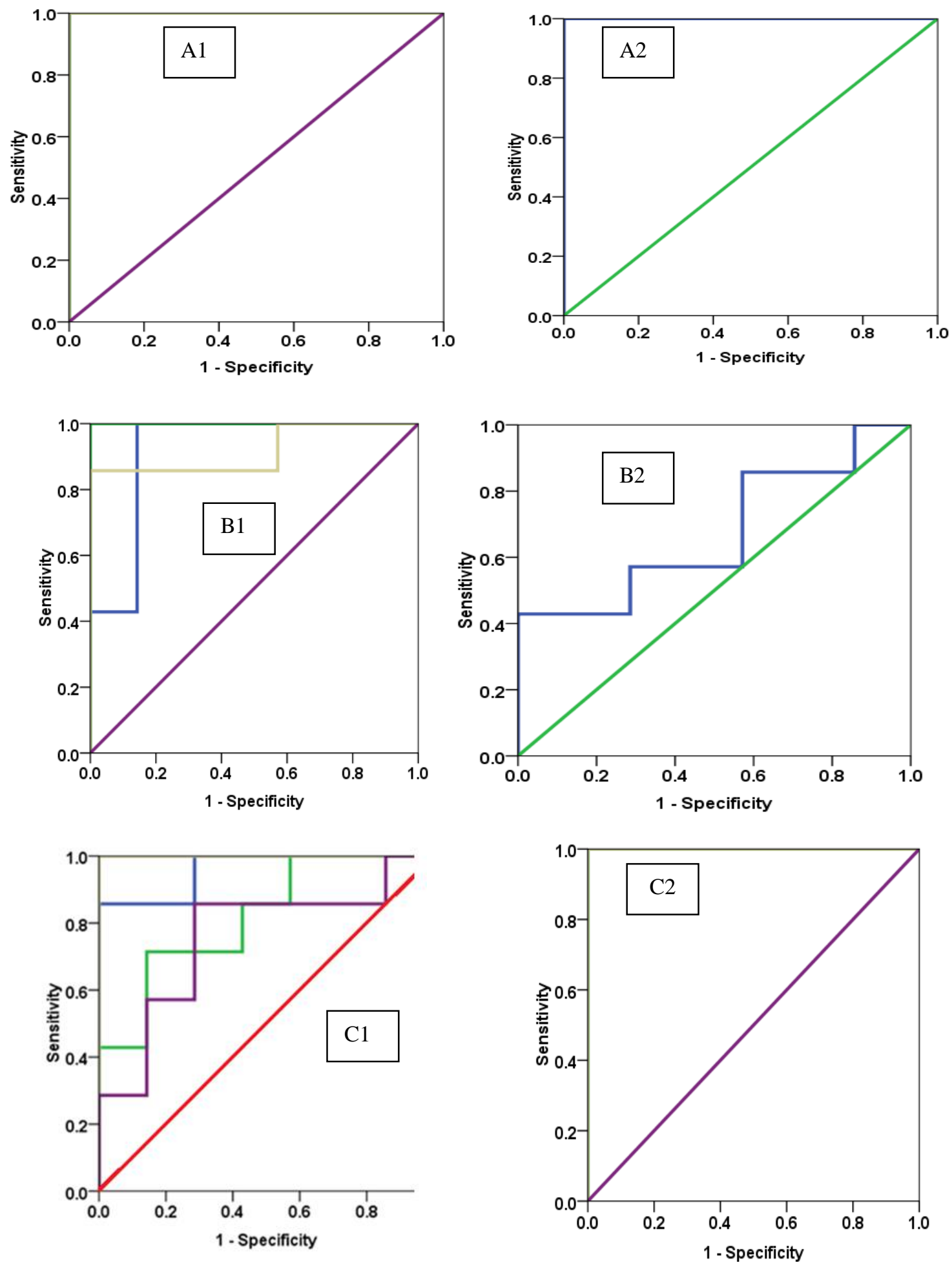

Figure 2: ROC Curve for all parameters in propinic acid group (A), ampicillin group (B) and ampicillinHSD fed group (C)

Table 4: Multiple regression using stepwise method for glutamate as a dependent variable

\begin{tabular}{lccccc}
\hline Predictor variable & Beta & $\boldsymbol{P}$-value & $\begin{array}{c}\text { Adjusted } \mathbf{R} \\
\text { square }\end{array}$ & F-value & Model \\
\hline Glut/Gln & -1.075 & 0.001 & 0.896 & 117.714 & 0.001 \\
Gln & 0.887 & 0.001 & 0.896 & \\
\hline
\end{tabular}


inhibits nerve growth and brain development. This inhibition often manifests as sugar cravings, hyperactivity, irritability, social impairment and cognitive disability [10].

Table 2 and Figure 1 show that PPA, ampicillin and a high-refined-sugar (sucrose) diet induce noticeable alterations in brain chemistry that manifest as a significant decrease or increase in almost all the measured neurotransmitters. This is related to the gut microbiota changes induced either by PPA, ampicillin or highly refined sugar during ampicillin treatment. This theory is supported by the recent study by Heijtz et al [11], who confirmed that after gut microbiota are disrupted by multiple factors including antibiotics, CNS chemistry is badly affected through the gutbrain axis.

Imbalances in the composition of gut microbiota in PPA or ampicillin-treated rats are observed as the overgrowth of $K$. pneumonia and $P$. vulgaris, which are propionibacteria and inducers of propionic acid producers, respectively. The concomitant alteration of most of the measured neurotransmitters together with the immune sequelae presented by the increase of IL-6, as shown in Table 2, may contribute to the development of persistent autistic features in treated rats [12].

The significant decrease in the concentration of NA in the three groups investigated (PPAtreated, ampicillin-treated and ampicillin-HSD fed) demonstrates the neurotoxic effects of PPA, ampicillin and HSD, as this neurotransmitter is essential for a wide range of CNS tasks and is also involved in neuronal growth [13]. NA has also been shown to limit neuroinflammation in the CNS [14]. This result can also be easily related to the significant elevation of IL-6, TNF $\alpha$, and IFN $\mathrm{y}$ in the brain homogenates of PPAtreated rats [4]. This could provide support for the contribution of PPA and ampicillin as environmental factors related to altered gutmicrobiota in the pathogenesis of autism.

The remarkable decrease in $5-\mathrm{HT}$ in the three groups studied can be easily related to the imbalance between inhibitory/excitatory transmission reported in autistic patients and rodent models of autism $[4,15]$. 5-HT interacts with DA, NE, GABA, and glutamate systems $[16,17]$ and is critical for the maturation of the GABA phenotype in the ventral spinal cord via 5HT1B receptors [16]. Furthermore, an interaction between the glutamate receptors mGLU5 and the 5-HT2A receptor was shown in mice [17]. The remarkable decrease of serotonin level in the brain of treated rats can be related to the toxic effects of PPA [3] or the association between microbiota and serotonin signaling. Both the ampicillin-treated and ampicillin-HSD fed groups demonstrated altered intestinal microbiota composition, which can easily relate to the remarkable decrease in serotonin [18].

Table 1 and Figure 1also demonstrate the significant decrease of the neurotransmitter DA in the three groups studied. This decrease can be attributed to the effects of PPA, ampicillin and ampicillin-HSD on DA transporters, thus increasing DA breakdown. This suggestion can find support in the work of Bello et al [19], who demonstrated that $20 \mathrm{~min}$ of access to $0.3 \mathrm{M}$ sucrose for only 6 days results in upregulation of the DA membrane transporters and downregulation of the DA receptors.

The significant increase in brain glutamate upon treatment with PPA can be easily related to ASD features. Glutamate excitotoxicity is one of the most important mechanisms involved in the etiology of autism. The elevation of glutamate, the main brain excitatory neurotransmitter, in the PPA, ampicillin-treated or ampicillin-HSD groups demonstrated excitotoxicity in the three groups studied. Elevation of glutamate can find support in a previous study [20], which reported that decreased GABA, an inhibitory neurotransmitter, could alter excitation/inhibition balance and lead to less inhibition of glutamatergic inputs in PPAtreated rat pups.

On the other hand, the significant increase of glutamate as a marker of excitotoxicity in ampicillin-treated animals contradicts a previous report by Rothstein et al. [21]. The report declared that several $\beta$-lactam antibiotics, to which ampicillin belongs, upregulate the expression of the glutamate transporter GLT-1 protein on astrocytes, thus reducing synaptic glutamate and contributing to decreased glutamate excitotoxicity. This inconsistency can be attributed to the substantial variation in the doses used. In the present study, $50 \mathrm{mg} / \mathrm{kg}$ body weight was used with the intention to alter gut microbiota and to study the effect of the induced overgrowth of PPA-related bacteria. The persistent elevation of glutamate in ampicillintreated rats fed a high-sucrose diet can be supported by the recent report by Kuang et al [22]. The study demonstrated that a maternal high-sucrose (HS) diet affects behavior and hippocampal neurons in the young offspring, leading to learning deficits through a significant increase in the expression of N-methyl-Daspartate (NMDA) receptors. These receptors serve as modulators of glutamate synaptic transmission, and thus excessive activation can 
reflect glutamate elevation and the disruption of calcium homeostasis, resulting in either apoptotic or necrotic neuronal death or both [23].

The significant increase of glutamine reported in the present study with the concomitant decrease of glutamate/glutamine ratio in PPA-treated rats can be supported by the recent study by Davison et al [24]. The authors performed neuroimaging using magnetic resonance spectroscopy (MRS) and demonstrated glutamine/glutamate elevation in the basal ganglia of all propionic acidemia patients during stable metabolic conditions. They attributed this elevation to the impact of hyperammonemia on cerebral brain water due to glutamine being osmotically active. The elevation of glutamine in ampicillin-treated or ampicillinHSD animals can be related to the altered microbiota, as presented in Table 1. Growth was detected in $K$. pneumonia, opportunistic pathogens known to generate ammonia in immune-compromised humans and animals, and hyperammonemia, which usually contributes to the pathogenesis of this bacterial species and clostridia as propionibacteria (not detected with the microbiological technique used in the present study) [25].

Table 3 shows the remarkable association between the impaired neurotransmitters and $1 \mathrm{~L}-$ 6. This association demonstrates that both neurochemistry alteration and neuroinflammation are involved in the neurotoxic effects of PPA and ampicillin. The results also confirms the toxic effect of HSD during antibiotic treatment.

The results demonstrate the satisfactory AUC, specificity and sensitivity of all measured parameters as markers for PPA, ampicillin and HSD.

\section{CONCLUSION}

The use of ampicillin influences the profile of microbiota through gut-brain interactions that affect brain neurochemistry and displays the risk of inducing biochemical autistic features. The remarkable effects of a high-sucrose diet on most of the measured parameters show that the induction of PPA-related microbial growth interacts with diet as an environmental risk factor.

\section{DECLARATIONS}

\section{Acknowledgement}

This research project was supported by a grant from the research division of the Female Center for Scientific and Medical Colleges at King Saud University.

\section{Conflict of Interest}

No conflict of interest associated with this work.

\section{Contribution of Authors}

The authors declare that this work was done by the authors named in this article and all liabilities pertaining to claims relating to the content of this article will be borne by them.

\section{Open Access}

This is an Open Access article that uses a funding model which does not charge readers or their institutions for access and distributed under the terms of the Creative Commons Attribution License (http://creativecommons.org/licenses/by/ 4.0) and the Budapest Open Access Initiative (http://www.budapestopenaccessinitiative.org/rea d), which permit unrestricted use, distribution, and reproduction in any medium, provided the original work is properly credited.

\section{REFERENCES}

1. Snavely SR, Hodges GR. The neurotoxicity of antibacterial agents. Ann Intern Med 1984; 101: 192104.

2. Adams JB, Johansen $L J$, Powell LD, Quig D, Rubin RA. Gastrointestinal flora and gastrointestinal status in children with autism--comparisons to typical children and correlation with autism severity. BMC Gastroenterol 2011; 11: 22.

3. MacFabe $D F$, Cain DP, Rodriguez-Capote $K$, Franklin $A E$, Hoffman JE, BoondF, Taylor AR, Kavaliers $M$, Ossenkopp KP. Neurobiological effects of intraventricular propionic acid in rats: possible role of short chain fattyacids on the pathogenesis and characteristics of autism spectrum disorders. Behav Brain Res 2007; 176: 149-169.

4. El-Ansary AK, Ben Bacha A, Kotb M. Etiology of autistic features: the persisting neurotoxic effects of propionic acid. J Neuroinflammation 2012; 9: 74.

5. Shaffer CL, Davey AM, Ransom JL, Brown YL, Gal P. Ampicillin-induced neurotoxicity in very-low-birth-weight neonates. Ann Pharmacother 1998; 32: 482-484.

6. Hopkins $M H$, Silverman RB. a-amino acid analogues as mechanism-based inactivators of $\gamma$-aminobutyric acid aminotransferase. Bioorg. Med. Chem. Lett 1992; 2(11): 1371-1374.

7. Alabdali A, Al-Ayadhi L, El-Ansary A. Association of social and cognitive impairment and biomarkers in autism spectrum disorders. J Neuroinflammation 2014; 11: 4 . 
8. Bayne K, Morris TH, France MP. Legislation and oversight of the conduct of research using animals: a global overview. In: The UFAW Handbook on the Care and Management of Laboratory and Other Research Animals (Hubrecht R, Kirkwood J, Eds), 123. Oxford: Wiley-Blackwell 2010; pp 107-123.

9. Zagrodzka J, Romaniuk A, Wieczorek M, Boguszewski P. Bicuculline administration into ventromedial hypothalamus: effects on fear and regional brain monoamines and GABA concentrations in rats: ActaNeurobiol. Exp 2000; 60: 333-343.

10. Dinan TG, Stilling RM, Stanton C, Cryan JF. Collective unconscious: How gut microbes shape human behavior. J Psychiat Res 2015; 63: 1-9.

11. Heijtz RD, Wang S, Anuar F, Qian $Y$, Björkholm B, Samuelsson A, Hibberd ML, Forssberg H, Pettersson S. Normal gut microbiota modulates brain development and behavior. Proc Natl Acad Sci USA 2011; 108(7): 3047-3052.

12. de Theije CG, Wopereis $H$, Ramadan M, van Eijndthoven $T$, Lambert J, Knol J, Garssen J, Kraneveld AD, Oozeer R. Altered gut microbiota and activity in a murine model of autismspectrum disorders. Brain Behav Immun 2014; 37: 197-206.

13. Clarke GL, Bhattacherjee A, Tague SE, Hasan W, Smith $P G . \beta$-adrenoceptor blockers increase cardiac sympathetic innervation by inhibiting autoreceptor suppression of axon growth. J. Neurosci 2010; 30(37): 12446-12454.

14. McNamee EN, Griffin EW, Ryan KM, Ryan KJ, Heffernan $S$, Harkin A, Connor TJ. Noradrenaline acting at betaadrenoceptors induces expression of IL-1beta and its negative regulators $I L-1 r a$ and $I L-1 R I I$, and drives an overall anti-inflammatory phenotype in rat cortex. Neuropharmacology 2010; 59(1-2): 37-48.

15. El-Ansary AK, Bacha AB, Ayahdi LY. Relationship between chronic lead toxicity and plasma neurotransmitters in autistic patients from Saudi Arabia. Clin Biochem 2011; 44(13): 1116-1120.

16. Allain AE, Segu L, Meyrand P, Branchereau P. Serotonin controls the maturation of the GABA phenotype in the ventral spinal cord via 5-HT1b receptors. Ann NY Acad Sci 2010; 1198: 208-219.

17. Halberstadt AL, Lehmann-Masten VD, Geyer $M A$, \& Powell SB. Interactive effects of mGlu5 and 5-HT2A receptors on locomotor activity in mice. Psychopharmacol (Berl) 2011; 215(1): 81-92.

18. Foster JA, McVey Neufeld KA. Gut-brain axis: how the microbiome influences anxiety and depression. Trends Neurosci 2013; 36 (5): 305-312.

19. Bello NT, Sweigart KL, Lakoski, JM, Norgren R, Hajnal A. Restricted feeding with scheduled sucrose access results in an upregulation of the rat dopamine transporter. Am. J. Physiol. Regul. Integr. Comp. Physiol 2003; 284(5): 1260-1268.

20. Van Nobelen NM, Kokkinidis L. Amygdaloid GABA not glutamate neurotransmission or mRNA transcription controls footshock-associated fear arousal inthe acoustic startle paradigm. Neuroscience 2006; 137(2): 707-716.

21. Rothstein JD, Patel S, Regan MR, Haenggeli C, Huang $Y H$, Bergles DE, Jin L, Dykes Hoberg M, Vidensky S, Chung DS, et al. Beta-lactam antibiotics offer neuroprotection by increasing glutamate transporter expression, Nature 2005; 433(7021): 73-77.

22. Kuang $H$, Sun M, Lv J, Li J, Wu C, Chen N, Bo L, Wei X, Gu X, Liu Z, et al. Hippocampal apoptosis involved in learning deficits in the offspring exposed to maternal high sucrose diets. J Nutr Biochem 2014; 25(9): 985990.

23. Gupta K, Hardingham GE, Chandran S. NMDA receptordependent glutamate excitotoxicity in human embryonic stem cell-derived neurons. Neurosci Lett 2013; 543: 95100.

24. Davison JE, Davies NP, Wilson M, Sun $Y$, Chakrapani A, McKiernan PJ, Walter JH, Gissen P, Peet AC. MR spectroscopy-based brain metabolite profiling in propionic acidaemia: metabolic changes in the basal ganglia during acute decompensation and effect of liver transplantation. Orphanet J Rare Dis 2011; 6: 19.

25. Kleiner $D$, Traglauer IA, DommS. Does ammonia production by Klebsiella contribute to pathogenesis? Bull Inst Pasteur 1998; 96: 257-265. 